\title{
Erratum: Using E-mail Communication Network for Importance Measurement in Collaboration Environments ${ }^{\star}$
}

\author{
Paweł Lubarski and Mikołaj Morzy \\ Institute of Computing Science \\ Poznan University of Technology \\ Piotrowo 2, 60-965 Poznan, Poland \\ Pawel.Lubarski@cs.put.poznan.pl, Mikolaj.Morzy@put.poznan.pl
}

\begin{abstract}
A. Nadamoto et al. (Eds.): SocInfo 2013 Workshops, LNCS 8359, pp. 43-54, 2014.
(C) Springer-Verlag Berlin Heidelberg 2014
\end{abstract}

DOI: $10.1007 / 978-3-642-55285-4 \_12$

In the initial version of the paper starting on page 43 of this volume, the authors Paweł Łupkowski and Paweł Kleka had been omitted. The correct affiliation should be:

Paweł Lubarski ${ }^{1}$, Paweł Łupkowski ${ }^{2}$, Paweł Kleka ${ }^{2}$, and Mikołaj Morzy ${ }^{1}$

${ }^{1}$ Institute of Computing Science

Poznan University of Technology

Piotrowo 2, 60-965 Poznan, Poland

Pawel.Lubarski@cs.put.poznan.pl, Mikolaj.Morzy@put.poznan.pl

${ }^{2}$ Department of Logic and Cognitive Science, Institute of Psychology,

Adam Mickiewicz University, Poznan, Poland

\{Pawel.Lupkowski, kleka\}@amu.edu.pl

* The original online version for this chapter can be found at: 10.1007/978-3-642-55285-4_4

A. Nadamoto et al. (Eds.): SocInfo 2013 Workshops, LNCS 8359, p. E1, 2016.

(C) Springer-Verlag Berlin Heidelberg 2016 